\title{
Non-randomness of the Full 4 and 5-pass HAVAL *
}

\author{
Hirotaka Yoshida ${ }^{1,2}$, Alex Biryukov ${ }^{2}$, Christophe De Cannière ${ }^{2 \star \star}$, \\ Joseph Lano ${ }^{2 \star \star \star}$, and Bart Preneel ${ }^{2}$ \\ 1 Systems Development Laboratory, Hitachi, Ltd., \\ 292 Yoshida-cho, Totsuka-ku, Yokohama, 244-0817, Japan \\ 2 Katholieke Universiteit Leuven, Dept. ESAT/SCD-COSIC \\ Kasteelpark Arenberg 10, B-3001 Leuven-Heverlee, Belgium \\ \{hyoshida, abiryuko, cdecanni, jlano, preneel\}@esat.kuleuven.ac.be
}

\begin{abstract}
HAVAL is a cryptographic hash function proposed in 1992 by Zheng, Pieprzyk and Seberry. Its structure is quite similar to other widely used hash functions such as MD5 and SHA-1. The specification of HAVAL includes a security parameter: the number of passes (that is, the number of times that a particular word of the message is used in the computation) which can be chosen equal to 3,4 or 5 . In this paper we cryptanalyze the compression functions of the 4-pass and the 5-pass HAVAL using differential cryptanalysis. We show that each of these two functions can be distinguished from a truly random function.
\end{abstract}

\section{Introduction}

A hash function is a cryptographic algorithm that takes input strings of arbitrary (or very large) length and maps these to short fixed length output strings. HAVAL is a cryptographic hash function proposed in 1992 by Zheng, Pieprzyk and Seberry [18]. Its structure is quite similar to other widely used hash functions such as MD5 [14] and SHA-1 [16]. It uses rotations, modular additions, and highly non-linear boolean functions. HAVAL operates in so called passes, where each pass consists of 32 steps. The recommended number of passes are 3, 4 and 5. Thus 3, 4 and 5 pass HAVAL would have 96, 128 and 160 steps (or rounds in block-cipher terminology) respectively. The hash value calculated by HAVAL is 256 bits long.

In the case of HAVAL, several articles demonstrated collisions for the reduced 2 -pass variants $[10,13,8]$. Recently, an efficient algorithm constructing collisions for the full 3-pass HAVAL has been described in [15]. The attack has a complexity of $2^{29}$ steps and requires a negligible amount of memory. However no weaknesses in the 4 and 5-pass HAVAL have been demonstrated so far.

* This work was supported in part by the Concerted Research Action (GOA) Mefisto2000/06 of the Flemish Government.

$\star \star$ F.W.O. Research Assistant, the Fund for Scientific Research - Flanders (Belgium).

$\star \star \star$ Research financed by a Ph.D. grant of the Institute for the Promotion of Innovation through Science and Technology in Flanders (IWT-Vlaanderen) 
In this paper we show a cryptanalysis of HAVAL in the cases where the number of passes equals the maximal security values: 4 and 5. Our analysis leads to an attack that detects the non-randomness of the 4-pass and the 5-pass HAVAL in encryption mode. We show how to distinguish the compression function of the 4 and 5-pass HAVAL from a random permutation. For convenience, we discuss the security of HAVAL focusing on the 4-pass version. Our discussion is easily extended to the 5-pass version. The security of the 4 and 5-pass HAVAL in hash mode remains an open problem.

The outline of this paper is as follows. In Section 2, we give a brief description of the HAVAL algorithm published in [18]. In Section 3, we present our differential attack on the HAVAL compression function used in encryption mode. We also discuss the practical implementation issues of our attack. In Section 4 we give experimental results. We conclude in Section 5.

\section{Description of the HAVAL Hash Function}

In this section, we give a brief description of the HAVAL hash function, which is sufficient to understand the concepts introduced in this paper. For a full description of HAVAL we refer to [18].

HAVAL is a hash function that is based on the well-known Davies-Meyer construction of hash functions ([12], p. 341). The variable-length message $M$ is divided into 1024 -bit blocks $M_{0}, M_{1}, \ldots, M_{n-1}$. The 256 -bit hash value $V_{n}$ is then computed as follows:

$$
V_{0}=I V ; V_{j+1}=\operatorname{compress}\left(V_{j}, M_{j}\right)=E\left(V_{j}, M_{j}\right)+V_{j} \text { for } 0 \leq j<n,
$$

where compress is the compression function, $I V$ is a fixed initial value and $E$ is a block cipher. As a block cipher $E$, one could choose either a known block cipher or a dedicated design. HAVAL chooses the latter option. The function $E$ is an iterated design that only uses simple operations on 32-bit words. The 256bit input $V_{j}$ is loaded into 8 registers $(A, B, C, D, E, F, G, H)$ and the 1024-bit message block is divided into 32 words of 32 bits $\left(X_{0} \ldots X_{31}\right)$.

The 8 registers are updated through a number of steps. One step of the compression function is depicted in Fig. 1. The HAVAL compression function consists of 96, 128 or 160 consecutive steps. Each sequence of 32 steps is grouped together into a pass, so that we say that HAVAL is 3,4 or 5-pass. In each pass, every word $X_{i}$ is used exactly once. Every pass $r$ has its own Boolean function $f_{r}$, 32 constants $K_{i}$, and a specified order in which the 32 words $X_{i}$ are processed.

\section{Differential Cryptanalysis of the 4-pass HAVAL in Encryption Mode}

In this section, we will explain the HAVAL hash function in encryption mode in Section 3.1 and will study known attacks on the reduced 2-pass and the full 3-pass HAVAL in Section 3.2. We will present a differential cryptanalysis to find 


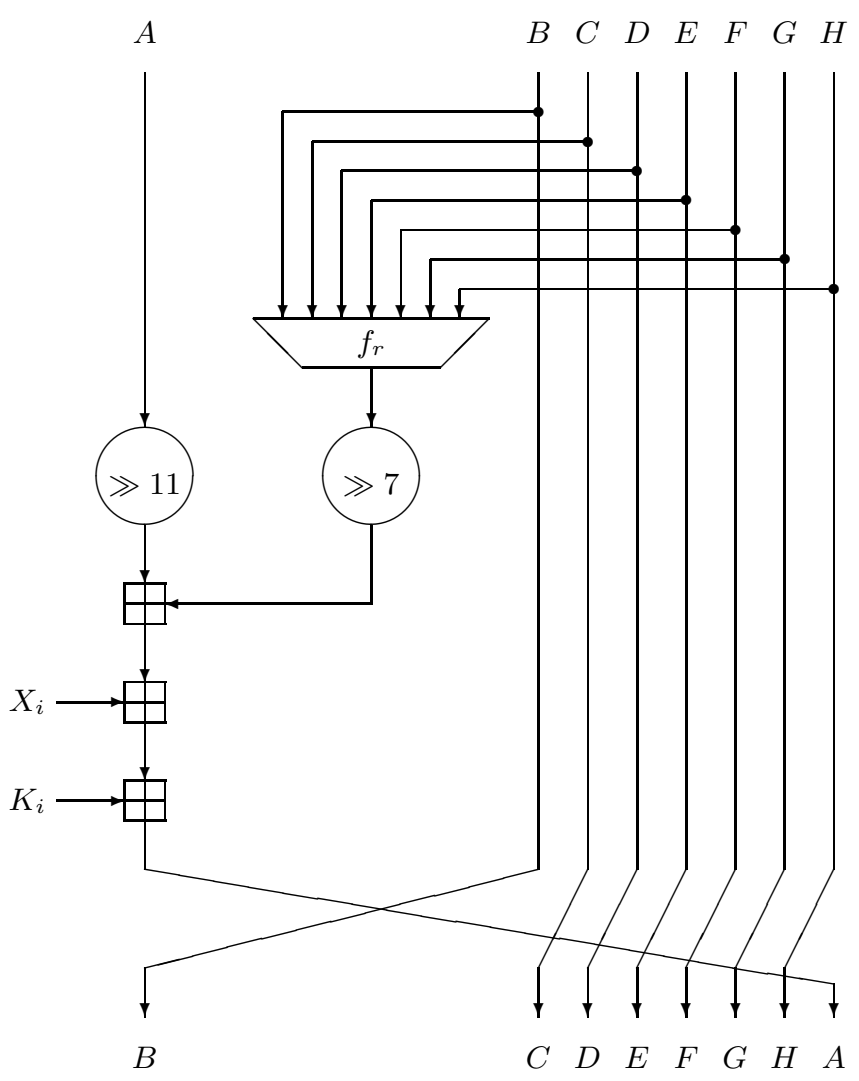

Fig. 1. One step of the compression function of HAVAL.

a weakness in the 4-pass HAVAL in Section 3.3 and provide solutions to the problems in its implementation in Section 3.4.

\subsection{Cryptanalysis of Hash Functions in Encryption Mode}

As mentioned above, one could construct a hash function from a block cipher using the Davies-Meyer construction. Inversely, one can construct a block cipher which is the HAVAL compression function with Davies-Meyer chaining peeled off. In the cipher, the message block $M_{j}$ is viewed as the key, the chaining variable $V_{j}$ acts as the plaintext block and $V_{j+1}=E\left(V_{j}, M_{j}\right)$ is the corresponding ciphertext block. In general, the block cipher constructed from a hash function in such a way is called a hash function in encryption mode.

We will analyze the 4-pass HAVAL in encryption mode. Such an analysis provides a better understanding of the strength of the HAVAL compression function. Our method can be easily extended to the 5 -pass version.

Several cryptanalytic techniques ranging from differential cryptanalysis [1] to slide attacks [2] have been applied to study the security of well-known hash 
functions in encryption mode. For example, differential cryptanalysis of SHA-1 has been shown in [7]. A slide attack on SHA-1 and an attack on MD5 which finds one high-probability differential characteristic were given in [17].

Throughout this paper we will use the notion of a "step", as defined in the specification of HAVAL, instead of the block-cipher notion - "round". We will also use the notion of a "pass", which stands for 32 steps as explained above.

\subsection{Known Attacks on the Reduced 2-Pass and the Full 3-Pass HAVAL}

In this section we review the previously known attacks which find collisions for the reduced 2-pass and the full 3-pass HAVAL and explain why the techniques in these attacks are not applicable to the 4-pass HAVAL.

The main idea in all the attacks of finding a collision is that the attacker uses the simplicity of the message schedule that allows him to control over the differences in the 8 registers. What is used is a pair of message whose difference with Hamming weight one is in exactly one message word. The difference in the message word is injected into registers at exactly one step in each pass.

In the attacks on the reduced 2-pass HAVAL which have two passes out of three, the difference injections explained above happen at two steps $[10,13]$. In between the two steps the difference in registers propagates. A differential propagation is found by applying the algebraic technique which is building a system of equations and solving it. The difference becomes zero at the last step in the propagation which means that a collision is found. When build the system, it is important to choose which message word have the difference. A good choice makes it possible for the difference in the registers to propagate for the small number of steps (e.g. 10), which allows the number of equations in the system to be small.

In the attack on the full 3-pass HAVAL, the algebraic technique as above is also applied [15]. The problem with this case is that the difference in the message word is injected into registers at three steps, such as 28, 38, and 69 . The attack solves this problem by combining the algebraic technique and differential cryptanalysis. From step 28 to 38 the algebraic technique is applied to find an inner almost collision, which means a pair of values in registers which differs only in the small number of bit positions and then from step 39 to 69 a differential cryptanalysis is to find a differential propagation with a high probability, $2^{-29}$ such that the difference is zero at the last step of the propagation. The attack indicates a weakness in the compression function against differential cryptanalysis.

In case of the full 4-pass HAVAL, we tried to apply the same strategy as the case of the full 3-pass, however it was turned out to be difficult to find an inner almost collision by applying the algebraic technique. This is due to the fact that the different order of processing message words from the order in the case of 3-pass makes the system of equation more difficult to solve. Even if an inner almost collision is constructed, a differential cryptanalysis has to solve the problem of finding a differential propagation of many steps (typically, 65) with 3 
time difference injection into the registers due to the message schedule, which is very difficult for the attacker to control over differences with a high probability. That is because the registers which the differences are injected from the message schedule into are not always as the attacker wants. We consider that it is easier to analyze the cipher in encryption mode where the differences in the register can be directly controlled than to do the cipher in hash mode where they can be controlled only through the message schedule. This observation above leads us to enhance differential cryptanalysis, instead of applying the algebraic technique, to find a weakness in the cipher in encryption mode.

\subsection{Differential Cryptanalysis of the 4-Pass HAVAL}

The technique of differential cryptanalysis has first been described in [1]. The aim of the approach is to find differential characteristics for the whole cipher. In [1], a differential characteristic is defined in the following:

Definition 1. Associated with any pair of encryptions are the difference of its two plaintexts, the differences of its ciphertexts, the differences of the inputs of each round in the two executions and the differences of the outputs of each round in the two executions. These differences form an n-round characteristic. A characteristic has a probability, which is the probability that a random pair with the chosen plaintext difference has the round and ciphertext differences specified in the characteristic.

In differential cryptanalysis, two difference operations are often used: $\Delta\left(X, X^{\prime}\right)=$ $X \oplus X^{\prime}, \Delta\left(X, X^{\prime}\right)=X-X^{\prime}$. We will consider both cases in our cryptanalysis.

The strategy to perform the differential cryptanalysis can be mainly divided into two parts: In the first part we divide the function into several consecutive sub-functions and try to find differential characteristics with high probability for these sub-functions. In our analysis, each sub-function will consist of several steps in a certain pass. Hence all steps in such a sub-function will use the same nonlinear Boolean function. In the second part the differential characteristics for each sub-function are concatenated so that they cover the whole cipher. However, it is difficult to do the second part of the analysis when the characteristics obtained in the first part have complicated forms. For instance, this is the case for SHA-1. In this paper, we present a method to solve this difficulty by combining these two parts into a single part.

The theoretical background of this method is the theory of Markov ciphers and their connection to differential cryptanalysis introduced by Lai et al. in [11]. For an iterated cipher $E$ with the function $Y=T(X, Z)$ which takes the plaintext input as $\mathrm{X}$, the subkey input as $\mathrm{Z}$, we denote the conditional probability that $\beta$ is the difference $\Delta Y(i)$ of the ciphertext pair after $i$ steps of $S$, given that $\alpha$ is the difference of the plaintext pair, by $P(\Delta Y(i)=\beta \mid \Delta X=\alpha)$.

Recall that a sequence of discrete random variables $v_{0}, v_{1}, \ldots, v_{r}$ is a Markov chain if, for $0 \leq i \leq r$,

$$
P\left(v_{i+1}=\beta_{i+1} \mid v_{i}=\beta_{i}, v_{i-1}=\beta_{i-1}, \ldots, v_{0}=\beta_{0}\right)=P\left(v_{i+1}=\beta_{i+1} \mid v_{i}=\beta_{i}\right) .
$$


A Markov chain is called homogenous if $P\left(v_{i+1}=\beta \mid v_{i}=\alpha\right)$ is independent of $i$ for all $\alpha$ and $\beta$. A Markov cipher is defined as follows:

Definition 2. An iterated cipher with the function $T$ is a Markov cipher if for all choices of $\alpha$ and $\beta$,

$$
P(\Delta Y=\beta \mid \Delta X=\alpha, X=\gamma)
$$

is independent of $\gamma$ when the subkey is uniformly random.

We now state the following theorem using our notation.

Theorem 1. If an r-step iterated cipher is a Markov cipher and the $r$ step keys are independent and uniformly random, then the sequence of differences $\Delta X=\Delta Y(0), \ldots, \Delta Y(r)=\Delta Y$, is a homogenous Markov chain.

In the case of HAVAL, we denote 8 consecutive steps ${ }^{3}$ of $E$ by $T$. We assume that the cipher $E$, obtained by iterating $T$, is a Markov cipher. This allows us to search for differentials rather than characteristics. The goal of our attack is to find a high probability differential for the 4-pass and the 5-pass HAVAL.

Our goal is to study differential properties of the 4-pass HAVAL. We will consider low Hamming weight differentials and their propagation: we study the behavior of the 4-pass HAVAL compression function when we apply input differentials of weight 1 and 2 . At the output, we only observe output differentials of weight 1 and 2 . We will check whether these observations are in accordance with the randomness criteria we would expect from a cryptographic hash function.

Let $\mathcal{A}$ be the set of all the bit strings of length 256:

$$
\mathcal{A}=\{0,1\}^{256} .
$$

Let $\mathcal{B}$ be the subset of $\mathcal{A}$ where each element has Hamming weight equal to 1 :

$$
\mathcal{B}=\{\Delta \in \mathcal{A} \mid \operatorname{Ham}(\Delta)=1\} .
$$

Let $\mathcal{C}$ be the subset of $\mathcal{A}$ where each element has Hamming weight equal to 2 :

$$
\mathcal{C}=\{\Delta \in \mathcal{A} \mid \operatorname{Ham}(\Delta)=2\} .
$$

Let $\mathcal{D}$ be the union set of $\mathcal{B}$ and $\mathcal{C}$ :

$$
\mathcal{D}=\mathcal{B} \cup \mathcal{C} .
$$

Let $\mathcal{E}$ be a set of integers where each element is greater than 0 and is less than or equal to the size of $\mathcal{D}$ :

$$
\mathcal{E}=\left\{1,2, \ldots, 2^{8}+\frac{2^{8} \cdot\left(2^{8}-1\right)}{2}\right\}
$$

\footnotetext{
${ }^{3}$ A single step of HAVAL is clearly a bad candidate for $T$ since only one 32-bit word changes per step and only one 32-bit word of key-material is mixed in.
} 
Using the first consecutive 8 steps in the $s$-th pass, we build a matrix $M_{s}$. To do so, we first define a function $g$ mapping $\mathcal{D}$ to $\mathcal{E}$ in the following manner. If $\Delta \in \mathcal{B}$, let $k$ be the position of 1 in $\Delta$. Otherwise, let $h$ be the high position of 1 in $\Delta$ and let $l$ be the low position of 1 in $\Delta$. The function $g$ is defined as follows:

$$
g(\Delta)= \begin{cases}k-1 & \Delta \in \mathcal{B} \\ h-l-1+\sum_{i=0}^{l-1}(256-i) & \Delta \in \mathcal{C} .\end{cases}
$$

It is easy to see that $g$ is bijective. The aim of the function $g$ is to establish an ordering for the elements of $\mathcal{D}$.

Now, let's denote the function which consists of the first consecutive 8 steps in the $s$-th pass as $T_{s}$. To construct a matrix $M_{s}$, we randomly choose a (sufficiently large) subset $R$ of $\mathcal{A}$. The cardinality of the subset $R$ is denoted by $\# R=r$. For $i$ and $j$ in $\mathcal{E}$, we define each entry $a_{i j}^{(s)}$ in the matrix $M_{s}$ as follows:

$$
a_{i j}^{(s)}=\frac{\#\left\{p \in R \mid g^{-1}(j)=\Delta\left(T_{s}(p), T_{s}\left(\Delta\left(p, g^{-1}(i)\right)\right)\right)\right\}}{r} .
$$

The entry $a_{i j}^{(s)}$ estimates the probability of the differential where the input difference is $g^{-1}(i)$ and the output difference is $g^{-1}(j)$. We assume that one pass of HAVAL behaves as a 4-round Markov cipher with $T_{s}$ as the round transformation $^{4}$. Thus the matrix $M_{s}$ is a transition matrix of the corresponding Markov chain. Raising this matrix to the fourth power as $M_{s}^{4}$ allows us to see the probabilities of 32 -step differentials for the $s$-th pass. Calculating the composition $\mu=\mu_{4}^{4} \circ \mu_{3}^{4} \circ \mu_{2}^{4} \circ \mu_{1}^{4}$ allows us to see the differential structure of the 4-pass HAVAL, where the function $\mu_{s}$ is defined by a matrix multiplication as $\mu_{s}(X)$ $=X \cdot M_{s}$. For example, we can see high probability differentials for the whole cipher. What is of most interest now is the highest value in the matrix $M=\mu(I)$. This highest value corresponds to a particular low-weight differential which has a high probability.

The approach described here has several complications with respect to a memory-efficient and fast implementation, which we will now explain into more detail.

\subsection{Implementing the Matrices $M_{s}$ and Their Multiplication}

We had to resolve some implementation issues for the $N \times N$ matrix $M_{s}$. The value of $N$ is quite large:

$$
N=2^{8}+\frac{2^{8} \cdot\left(2^{8}-1\right)}{2} \approx 2^{15}
$$

\footnotetext{
${ }^{4}$ Our experiments indicate that this assumption is reasonable. The ten best differentials for 16 steps produced by experiment and the ten best differentials computed via $M_{s}^{2}$ were at most a factor 1.28 apart. Also some variation across the different keys has been observed.
} 
If we implement the matrix $M_{s}$ as such, the required memory size is $8 \cdot 2^{30} \approx 8 G B$ when using 64-bit variables to represent each element of $M_{s}$. This is quite large and not efficient at all. Simulations show that the matrix $M_{s}$ is very sparse: Because of the diffusion of $T_{s}$, the hamming weight of the output differences are very likely to be more than 2 and thus most output differences will be discarded in our approach. And those that do have a Hamming weight of at most 2 only occur in a limited number of places in the matrix. This helps us for the efficient implementation. The number of nonzero entries of a row in a matrix is typically 100 but we make the number a parameter, namely $d$, which is useful as will be shown. For each row, we will now only store the nonzero entries, together with the column in which this nonzero entry occurs. Furthermore, we also store the row itself for each row. This is useful to reduce the time complexity which will be explained later. Every non-zero $a_{i j}^{(s)}$ in the $M_{s}$ is stored as a triplet $\left(i, j, a_{i j}^{(s)}\right)$ in its implementation. In that case, the memory complexity is only $2^{15} \cdot d \cdot(2+2+8) \approx 5 M B$ when using 16-bit variables to represent each row $i$ and column $j$.

In order to implement the multiplication of the two matrices $M_{s}$, we have to implement one matrix $M_{s}$ as a list of rows and the other matrix as a list of columns. The representation of $M$ as a list of columns can be easily obtained from the representation as a list of rows by a straightforward and efficient transposition algorithm. In addition to the memory for the two lists, the memory for the product matrix is allocated. However, we have a problem with the memory for the product matrix after one multiplication. A theoretical estimation shows that about $26 \%$ of the entries in the product matrix will be non-zero, which was confirmed by our experiments. This means that the product matrix is not sparse any more. This motivates the following idea of pruning the matrix. After the generations or multiplications of the matrix $M_{s}$, we only keep high-value entries in $M_{s}$, cutting the entries below a fixed low value $q$. To obtain a high probability for the whole cipher, it is sufficient to obtain high probabilities after every 8 steps. This also motivates the idea of pruning. We now keep the matrix sparse all the steps. Taking into account that the multiplications can be done on the fly, the memory complexity of computing the matrix $M$ for the whole cipher is $15 M B$.

The time complexity of generating the matrix $M_{s}$ can be shown to be $r \cdot 2^{16}$ computations of $T_{s}$. When we take $r$ equal to $2^{20}$ (which seems to be enough to obtain a matrix with sufficient statistical significance), this results in a time complexity of $2^{36}$ computations of $T_{s}$. As for the multiplication of the matrices, we can find a fast implementation. As mentioned above, the matrix $M_{s}$ is implemented as an array with dimension two where each element is a triplet $\left(i, j, a_{i j}^{(s)}\right)$. If the matrix is sorted by the first element and afterwards by the second element in the triplets, then it can be implemented as an array with dimension one. Now all we have to do is the multiplication of two sorted arrays with dimension one. In this case, the multiplication of the matrices has a time complexity of $N \cdot d=2^{15} \cdot 100 \approx 2^{21.6}$ computations, each of which is a few of multiplications and additions of two 64 -bit variables. Note that this significantly 
reduces the number of computations of entries during the multiplication of the matrices hence is much faster than straightforward implementation which has a time complexity $N^{2} \cdot d^{2} \approx 2^{43.3}$. To compute the matrix $M$ for the whole cipher requires the generations of the matrix $M_{s} 5$ times and the multiplication of matrices 15 times. Taking into account their time complexities and what is done during one computation in each case, the time complexity in total is dominated by the former, which is $2^{38}$ computations of $T$.

\section{Experimental Results}

We report some experimental results which we obtained by using the techniques described above. In our experiments, we used the reference implementation of HAVAL available at [9] and the cut-away value $q$ in Section 3.4 is set to be $2^{-12}$. Since different passes use different non-linear functions, it is interesting to see the differential structure for each of the 4 different passes. Therefore we search for the best probability over 8 and 32 steps in the $s$-th pass.

Before doing that, we need some preparation. We focus on the observation of 8 steps to see whether the experimental results are stable. That is very important: if the results were not stable yet, the error will be amplified due to the multiplication of matrices. Our simulations convincingly showed that increasing the number of plaintexts $r$ to $2^{20}$ is sufficient in order to obtain precise experimental results.

Table 1 presents a typical experimental results that we observed for the 4Pass HAVAL. It shows the measured probability for 8 steps for each of the 4 passes, and this for increasing samples $r$. As a difference function we used $\Delta\left(X, X^{\prime}\right)=X \oplus X^{\prime}$. Note that in the above experiment, we consider the input difference $\Delta_{i n} \in \mathcal{B}$ to make it feasible in terms of time complexity to obtain the result for $r$ equal to $2^{24}$. However, in all the following experiments we will use the entire set $\mathcal{D}$.

Table 1. The best probability for 8 steps in the case of $\Delta\left(X, X^{\prime}\right)=X \oplus X^{\prime}$

\begin{tabular}{|c|c|c|c|}
\hline & $r=2^{16}$ & $r=2^{20}$ & $r=2^{24}$ \\
\hline$s=1$ & $2^{-5.916521}$ & $2^{-5.992885}$ & $2^{-5.996883}$ \\
$s=2$ & $2^{-6.947432}$ & $2^{-7.023257}$ & $2^{-7.009951}$ \\
$s=3$ & $2^{-6.969333}$ & $2^{-6.996482}$ & $2^{-6.992622}$ \\
$s=4$ & $2^{-6.594859}$ & $2^{-6.660011}$ & $2^{-6.677051}$ \\
\hline
\end{tabular}

Table 2 shows the best probability of a differential characteristic for all 4 passes $(s=1,2,3,4)$, and this both for 8 steps and 32 steps. In these experiments we used $2^{20}$ as a value for $r$, and again used the difference function $\Delta\left(X, X^{\prime}\right)=$ $X \oplus X^{\prime}$ 
Table 2. The best probability for 8 and 32 steps in the case of $\Delta\left(X, X^{\prime}\right)=X \oplus X^{\prime}$

\begin{tabular}{|c|c|c|}
\hline & 8 steps & 32 steps \\
\hline$s=1$ & $2^{-5.992885}$ & $2^{-26.670829}$ \\
$s=2$ & $2^{-7.023257}$ & $2^{-30.992364}$ \\
$s=3$ & $2^{-6.996482}$ & $2^{-30.908851}$ \\
$s=4$ & $2^{-6.660011}$ & $2^{-29.360996}$ \\
\hline
\end{tabular}

For both the 4-pass and the 5-pass HAVAL, we can now calculate the best probability with which all the differential characteristics we consider by computing the matrix $M$ in Section 3 in which the highest entry is the best probability. We learn from Section 3 that it is practical to compute the matrix $M$ because the time complexity is $2^{38}$ computations of 8 steps and the memory complexity is $15 M B$.

The result is that the best probability is $2^{-125}$ for the 4-pass HAVAL and $2^{-168}$ for the 5-pass HAVAL. Each of these two probabilities is much greater than the probability $2^{-256}$ that we would expect from a truly random hash function. This means that both the 4-pass and the 5-pass HAVAL have a significant weakness of randomness.

Taking into account that each of these our results has been obtained with a fixed randomly chosen key, there could be an occupancy problem if our results are affected by the choice of the key. There are two points to be stressed:

For the full 4 and 5-Pass HAVAL we fix both the input and the output differences. Thus occupancy is not a problem there. On the other hand we gather probabilities for the matrix $M$ by experiment, so we could potentially experience occupancy problem, which would result in slightly higher key-dependent probabilities.

We carried out some experiments for the 8 steps of each pass which show that probabilities collected for the matrix $M$ are not key-dependent and hold on average. We encrypted $2^{20}$ plaintext pairs to check that results of Table 2 for 8 steps remain the same for 5 different keys $K_{1}, K_{2}, \cdots, K_{5}$. The following table 3 shows the best probabilities over different keys. The table 3 shows that

Table 3. The best probabilities over different keys

\begin{tabular}{|c|c|c|c|c|}
\hline Key & 1st pass & 2nd pass & 3rd pass & 4th pass \\
\hline$K_{1}$ & $2^{-5.992885}$ & $2^{-7.012381}$ & $2^{-6.972267}$ & $2^{-6.647129}$ \\
$K_{2}$ & $2^{-5.979194}$ & $2^{-6.977285}$ & $2^{-6.997537}$ & $2^{-6.651963}$ \\
$K_{3}$ & $2^{-5.974168}$ & $2^{-6.980409}$ & $2^{-7.007947}$ & $2^{-6.668383}$ \\
$K_{4}$ & $2^{-5.984237}$ & $2^{-6.979541}$ & $2^{-7.005823}$ & $2^{-6.676664}$ \\
$K_{5}$ & $2^{-5.990521}$ & $2^{-6.983540}$ & $2^{-6.972958}$ & $2^{-6.682163}$ \\
\hline
\end{tabular}


results of Table 2 for 8 steps remain the same for these keys, which means the best probabilities are not affected by the choice of the key.

Next we consider not only the probabilities and but also their pairs of input difference and output difference to see if the differential structure of 8 steps is affected by the choice of the key. In fact, in the case of the 2nd pass, the best probabilities in the table 3 are achieved at exactly the same pair of input difference and output difference. In the cases of the other passes, the best probabilities in the table 3 are achieved at several different pairs of input and output differences. Therefore we present for each pass, the probabilities over different keys at the same pair of input and output difference in the table 4 in which each triplet where the probability is not the best is indicated by \#. Fortunately, the probabilities for each pass are very close which means the differential structure of 8 steps is not affected by the choice of the key.

Table 4. The high probabilities at the same pair of input difference and output difference for each pass

\begin{tabular}{|c|c|c|c|c|}
\hline Key & 1st pass & 2nd pass & 3rd pass & 4th pass \\
\hline$K_{1}$ & $2^{-5.992885}$ & $2^{-7.012381}$ & $2^{-6.972267}$ & $2^{-6.647129}$ \\
$K_{2}$ & $\# 2^{-5.997537}$ & $2^{-6.977285}$ & $\# 2^{-7.010606}$ & $2^{-6.651963}$ \\
$K_{3}$ & $2^{-5.974168}$ & $2^{-6.980409}$ & $\# 2^{-7.009896}$ & $\# 2^{-6.687684}$ \\
$K_{4}$ & $\# 2^{-5.988511}$ & $2^{-6.979541}$ & $2^{-7.005823}$ & $\# 2^{-6.686834}$ \\
$K_{5}$ & $\# 2^{-6.000352}$ & $2^{-6.983540}$ & $\# 2^{-7.015938}$ & $\# 2^{-6.684284}$ \\
\hline
\end{tabular}

These discussions show that our results hold on average for any key and are not affected by the occupancy problem.

Our method contains multi-paths. This means that various trails exist that go from one input difference to one output difference. Therefore an interesting question is how many multi-paths are included into the best probability. We carried out some experiments with $2^{20}$ plaintexts and found an answer to the question. The best probability $2^{-124.6}$ with which the input difference $e_{160}$ goes to the output difference $e_{176}$ includes 12 multi-paths. On the other hand, a probability $2^{-125.9}$ with which the input difference $e_{139}$ goes to the output difference $e_{155}$ includes the maximum number of multi-paths observed, 42. For both probabilities above, the input and output difference have Hamming weight 1.

Another point we need to check is which difference notion is most effective in our attack: $\Delta\left(X, X^{\prime}\right)=X-X^{\prime}$ or $\Delta\left(X, X^{\prime}\right)=X \oplus X^{\prime}$. In Table 5 , we present the results for the case of the difference operation $\Delta\left(X, X^{\prime}\right)=X-X^{\prime}$, where the number of samples $r$ equals $2^{16}$. The table shows the best probability over 8 and 32 steps in the $s$-th pass for the 4-Pass HAVAL. By comparing this table with Table 2, we can see that the difference operation $\Delta\left(X, X^{\prime}\right)=X \oplus X^{\prime}$ is more effective. One of the possible reasons why this happens is that due to the nonlinear function, each step of the 4-Pass HAVAL uses XOR operation 4 to 8 times 
while it uses arithmetic additions only 3 times. This makes the step function XOR-friendly which means that differences can go though paths with paying relatively small probabilities when using the operation. $\Delta\left(X, X^{\prime}\right)=X \oplus X^{\prime}$.

Table 5. The best probability for 8 and 32 steps in the case of $\Delta\left(X, X^{\prime}\right)=X-X^{\prime}$

\begin{tabular}{|c|c|c|}
\hline & 8 steps & 32 steps \\
\hline$s=1$ & $2^{-6.860449}$ & $2^{-27.638838}$ \\
$s=2$ & $2^{-7.426353}$ & $2^{-34.011164}$ \\
$s=3$ & $2^{-7.642448}$ & $2^{-34.337646}$ \\
$s=4$ & $2^{-7.536476}$ & $2^{-30.437003}$ \\
\hline
\end{tabular}

Note that these results can not be used immediately to distinguish outputs of the 4-Pass or the 5-Pass HAVAL in hash mode from truly random outputs, though they show a surprising property.

We explain what we have done in details and what we will be able to do for the future research. We limited ourselves to search for all the paths where the differences have a hamming weight less than 3 not only at input and output but also at every 8 steps, which is a strong condition on the paths. It is surprising to find a path with a very good probability under this limited circumstance. This means that we found a probability which is a lower bound for the differentials. It would be interesting to see how high the best probability be when the condition is relaxed. In order to do this, a more efficient algorithm has to be found.

We describe what would be necessary for a hash function which is secure against our attack. One of the necessary conditions to apply our attack is that the weight of a low-weight difference is likely to remain to be low after 8 steps. This is the case for the 4-Pass and the 5-Pass HAVAL. For a hash function with a good diffusion, this is not the case even after a small number of consecutive steps. Our attack is not applicable to such a function.

\section{Conclusions}

We have analyzed the compression functions of the 4-pass and the 5-pass HAVAL. Surprisingly, our result shows that the use of highly non-linear functions, which is the main focus of the design of HAVAL, does not result in a hash function which is significantly strong against differential cryptanalysis. With our approach, we identified differentials with probabilities $>2^{-125}$ for the 4-pass HAVAL and $>2^{-168}$ for the 5 -pass HAVAL, which is much higher than the probability $2^{-256}$ we would expect from a random function.

It is difficult to see if and how the weakness of randomness in the compression function can be exploited to find collisions for the HAVAL hash function. This remains an open problem. The strategy for our attack is quite general so that we can analyze the compression functions of other hash functions with the approach described in this paper. 


\section{Acknowledgements}

The authors would wish to thank Bart Van Rompay, Antoon Bosselaers, Soichi Furuya, Souradyuti Paul, and several anonymous reviewers for helpful comments and useful discussions.

\section{References}

1. E. Biham, A. Shamir, Differential Cryptanalysis of the Data Encryption Standard, Springer-Verlag, 1993.

2. A. Biryukov, D. Wagner, Advanced slide attacks, Eurocrypt 2000, LNCS 1807, B. Preneel, Ed., Springer-Verlag, pp. 589-606, 2000.

3. B. den Boer, A. Bosselaers, Collisions for the compression function of MD5, Eurocrypt 1993, LNCS 765, T. Helleseth, Ed., Springer-Verlag, pp. 293-304, 1993.

4. I. Damgård, A design principle for hash functions, Crypto 1989, LNCS 435, G. Brassard, Ed., Springer-Verlag, pp. 416-427, 1990.

5. H. Dobbertin, The status of MD5 after a recent attack, Cryptobytes, Vol. 2, No. 2, pp. 1-6, Summer 1996.

6. H. Gilbert, H. Handschuh, Security Analysis of SHA-256 and Sisters, SAC 2003, LNCS 3006, M. Matsui, R. Zuccherato, Eds., Springer-Verlag, pp. 175-193, 2004.

7. H. Handschuh, D. Naccache, SHACAL, Submission to the NESSIE project, 2000. Available from http://www.gemplus.com/ smart/r_d/publications/pdf/HNOOshac.pdf.

8. Y.-S. Her, K. Sakurai, S.-H. Kim, Attacks for finding collision in reduced versions of 3-pass and 4-pass HAVAL, International Conference on Computers, Communications and Systems, CE-15, pp. 75-78, 2003.

9. Calyptix Security, HAVAL source code (reference implementation), available at http://www.calyptix.com/downloads.html.

10. P. Kasselman, W. Penzhorn, Cryptanalysis of reduced version of HAVAL, Electronics letters, Vol. 36, No. 1, pp. 30-31, January 2000.

11. X. Lai, J. Massey, Markov Ciphers and Differential Cryptanalysis, Eurocrypt 1991, LNCS 547, D. Davies, Ed., Springer-Verlag, pp. 17-38, 1991.

12. A. Menezes, P. van Oorschot and S. Vanstone, Handbook of Applied Cryptography, CRC Press, 1997.

13. S. Park, S. H. Sung, S. Chee, J. Lim, On the security of reduced versions of 3-pass HAVAL, ACISP 2002, LNCS 2384, J. Seberry, L. Batten, Eds., pp. 406-419, 2002.

14. R. Rivest, The MD5 message-digest algorithm, Request for Comments (RFC) 1321, Internet Activities Board, Internet Privacy Task Force, April 1992.

15. B. van Rompay, A. Biryukov, B. Preneel, J. Vandewalle, Cryptanalysis of 3-Pass HAVAL, Asiacrypt 2003, LNCS 2894, C. Laih, Ed., Springer-Verlag, pp. 228-245, 2003.

16. National Institute of Standards and Technology, FIPS-180-2: Secure Hash Standard (SHS), August 2002.

17. M. Saarinen, Cryptanalysis of Block Ciphers Based on SHA-1 and MD5, FSE 2003, LNCS 2887, T. Johansson, Ed., Springer-Verlag, pp. 36-44, 2003.

18. Y. Zheng, J. Pieprzyk, J. Seberry, HAVAL - a one-way hashing algorithm with variable length of output, Auscrypt 1992, LNCS 718, J. Seberry, Y. Zheng, Eds., Springer-Verlag, pp. 83-104, 1992. 